\title{
Simulation of loss-of-flow transients in research reactors
}

\author{
Christos Housiadas \\ “Demokritos" National Centre for Scientific Research, P.O. Box 60228, 15310 Agia Paraskevi, Athens, Greece.
}

\begin{abstract}
The course of loss-of-flow transients in pool-type research reactors, with scram disabled, is investigated. The analysis is performed with a customized version of the code PARET. The focus is on determining the two-phase flow stability boundaries as function of initial reactor conditions, recognizing that flow instability is the basic mechanism responsible for core damage in such type of transients. A useful chart is provided, which describes the stability region in terms of initial reactor power, initial pool temperature, peaking factor, and flow-decay time constant.
\end{abstract}

Keywords: Research reactors, loss-of-flow accident, flow instabilities, PARET.

\section{Introduction}

In the past decade much effort has been invested in the safety re-assessment of existing research reactors, as stemmed from the worldwide campaign of converting research reactor fuel from Highly Enriched Uranium (HEU) to Low Enriched Uranium (LEU). Most of the work performed can be found in a special five-volume issue of the International Atomic Energy Agency (IAEA), which reports in detail the safety-related calculations conducted in various institutions throughout the world in response to a benchmark exercise on computational methods (IAEA, 1992).

The research reactor safety calculations entail usually the simulation of several selected cases within two broad accident categories, namely, reactivity insertion accidents, and loss-of-flow accidents (LOFAs). In the analysis of reactivity accidents it is customary to consider systematically transients with and without scram event, called respectively, protected transients and unprotected or self-limited transients. Unprotected reactivity transients have received prompt (Woodruff, 1984), as well as recent attention (Nasir et al., 1999; Mirza et al., 1998) because of their relevance in determining the reactivity insertion limits imposed by clad melting temperature. In contrast, the LOFA simulations have been limited so far to the investigation of protected transients only. With the reactor shutdown system enabled, all LOFA simulations predict that clad temperature remains well below the clad melting point and that no flow instabilities take place in the cooling channels (IAEA, 1992). On the other hand, research reactor operators miss any data on reactor power and clad temperature responses in case of an unprotected event.

The objective of the present work is to investigate through numerical simulations the course of unprotected loss-of-flow transients in a typical pool-type research reactor. The applicable accident sequences are those anticipating failure of reactor scram in occurrences like primary pump failure, valve failure, blockage in piping or heat exchanger, pump unavailability due to loss of electrical power etc. Under such circumstances, it is generally proposed to assume that partial core damage occurs (IAEA, 1986). The present work examines the conservatism of this assumption. This is done by investigating the occurrence (or not) of two-phase flow instability during the transient, which as discussed next, is the precursor mechanism for plate damage.

Flow instabilities in boiling channels have been extensively investigated because of their relevance to BWRs and power industry in general (e.g. Achard et al., 1985; Saha et al., 1976; Yadigaroglu and Bergles, 1972). Most of the work has been done in the area of the so-called dynamic or density-wave instabilities, usually manifested in high-pressure systems. Low-pressure systems like research reactors are susceptible to a different form of instability known as flow excursion or Ledinegg instability (Hu and Bernard, 1999; Oh and Chapman, 1996). This instability, which is due to a negative slope in the pressure drop versus flow-rate curve, can be described as follows. In the occurrence of massive vapor generation, liquid phase is forced to accelerate to compensate for the large flow area reduction (gas-to-liquid specific volume ratio is very high at low pressure). This creates a significant pressure drop, thereby lowering flow-rate through the channel, which in turn leads to more vapor generation. As a result, clad surface may experience premature burnout, as indeed has been observed in 
experimental investigations (Whittle and Forgan, 1967). Instead, if the transient is free of instabilities, then one may reasonably anticipate that the accident, even unprotected, will be self-terminated due to reactor feedback, without compromising core integrity. Based on this remark, the analysis focuses on determining the stability boundaries and their sensitivity to relevant operational and physical parameters. The simulations are performed with the help of code PARET (Woodruff, 1984; Obenchain, 1969), which is a widely used state-of-the-art calculational tool for carrying out coupled neutronic, thermal-hydraulic analyses in research reactors. The results effectively confirmed the existence of a large band of conditions for which core safety is maintained during the transient.

The present work will help in improving the assumptions made in the event sequences analysis, as required by the current methodologies for research reactor safety assessment (IAEA, 1994). The results may also help in setting appropriate operational limits ensuring safe reactor response in unprotected LOFA events. On the other hand, the analysis can be of interest to Probabilistic Safety Assessment (PSA) methods. There is an increasing trend in using PSA methods as a supplement to the conventional deterministic approaches in research reactor safety. An important issue is to evaluate without excessive conservatism the frequency of the events leading to core damage. Hence, it is of interest to dispose means to assess relevant event sequences such as unprotected LOFAs.

The work evolves in the following way: section 2 gives a short outline of the Greek Research Reactor-1 (GRR-1), selected as the reference design. Section 3 describes the modelling approach, while section 4 presents the results of the simulations. Finally, the conclusions are given in section 5.

\section{Reactor description}

The Greek Research Reactor-1 (GRR-1) is considered as the reference design in the present investigation. It is a 5 MW pool-type reactor with MTR-type fuel elements, cooled and moderated with demineralized light water.

In line with the international Reduced Enrichment for Research and Test Reactor (RERTR) programme, the core has been recently fuelled with LEU elements of $U_{3} \mathrm{Si}_{2}-\mathrm{Al}$ type. The fuel enrichment is $19.75 \%$ and the fissile loading is $12.34 \mathrm{~g}$ of ${ }^{235} \mathrm{U}$ per plate. The equilibrium LEU core contains 28 standard fuel elements and 5 control fuel elements, arranged on a $6 \times 9$-element grid plate. Each standard fuel element consists of 18 flat plates. The control fuel element is of the same size as the standard element but consists of only 10 plates, thus providing an inner gap for the insertion of the control blades. The control material is composed of $\mathrm{Ag}(80 \%), \mathrm{Cd}(5 \%)$ and $\mathrm{In}$ $(15 \%)$. The core is reflected by Beryllium on two opposite faces and is surrounded by a practically infinite thickness of pool water. One graphite thermal column is adjacent to one side of the core. In the middle of the core there is a flux trap.

The core is suspended in a water pool that is $9 \mathrm{~m}$ deep, having a volume of approximately $300 \mathrm{~m}^{3}$. The fuel elements are cooled by circulating the water of the pool at a rate of $450 \mathrm{~m}^{3} / \mathrm{h}$. The water flows downward through the core, passes through a decay tank and then pumped back to the pool through the heat exchangers. A weighted flapper valve attached to the bottom of the core exit plenum enables natural circulation through the core in the absence of forced flow circulation. Core inlet temperature, i.e. pool water, is not permitted to exceed $45^{\circ} \mathrm{C}$. Pool temperature depends on reactor power, as well as external temperature, because the latter affects heat dissipation in the cooling towers. Figure 1 shows pool water temperature measurements, as recorded over several months of reactor operation. As can be seen, core inlet temperature (thermocouple A) varies in the range between $20^{\circ} \mathrm{C}$ and $44^{\circ} \mathrm{C}$. Also note that quite homogeneous temperature conditions prevail in the pool, considering that similar measurements are obtained from two distant thermocouples.

Table 1 summarizes the reference design data. It can be noticed that the majority of material test and research reactors are characterized by similar design parameters. Hence, the selected data can be regarded as typical of pool-type research reactors.

\section{Modelling}

The simulations have been performed with the code PARET, which employs a one-dimensional thermalhydraulic model, coupled with a point reactor kinetic model. The code was originally developed at the Idaho National Engineering Laboratory for conditions typical of power reactors (Obenchain, 1969) and later adapted at the Argonne National Laboratory for the analysis of transient behaviour in research reactors (Woodruff, 1984). 
Table 1. Reference design data

\begin{tabular}{ll}
\hline Reactor & Pool-type \\
Power level $(\mathrm{MW})$ & 5 \\
Grid plate & $6 \times 9$ \\
Fuel & $\mathrm{U}_{3} \mathrm{Si}_{2}$-Al \\
Fuel enrichment $(\mathrm{wt} \%)$ & 19.75 \\
Standard fuel elements & 28 \\
Control fuel elements & 5 \\
Water boxes & 1 \\
Flux trap & 1 (central) \\
Reflector & Beryllium \\
Fuel plates per element & \\
$\quad$ Standard & 18 \\
$\quad$ Control & 10 \\
Plate dimensions $(\mathrm{mm})$ & $66.6 \times 1.52 \times 625.5$ \\
Fuel meat dimensions $(\mathrm{mm})$ & $62.3 \times 0.50 \times 609.5$ \\
Fissile loading $\left(\mathrm{g}{ }^{235} \mathrm{U} / \mathrm{plate}\right)$ & 12.34 \\
Uranium density in fuel meat $\left(\mathrm{g} / \mathrm{cm}^{3}\right)$ & 3.36 \\
Clad material, thickness $(\mathrm{mm})$ & $\mathrm{Al}, 0.51$ \\
Water channel thickness $(\mathrm{mm})$ & 2.90 \\
Coolant flow rate $\left(\mathrm{m}^{3} / \mathrm{h}\right)$ & 450 \\
Coolant inlet temperature $\left({ }^{\circ} \mathrm{C}\right)$ & $20 \div 44$ \\
Coolant inlet pressure $($ bar $)$ & 1.796 \\
\hline
\end{tabular}

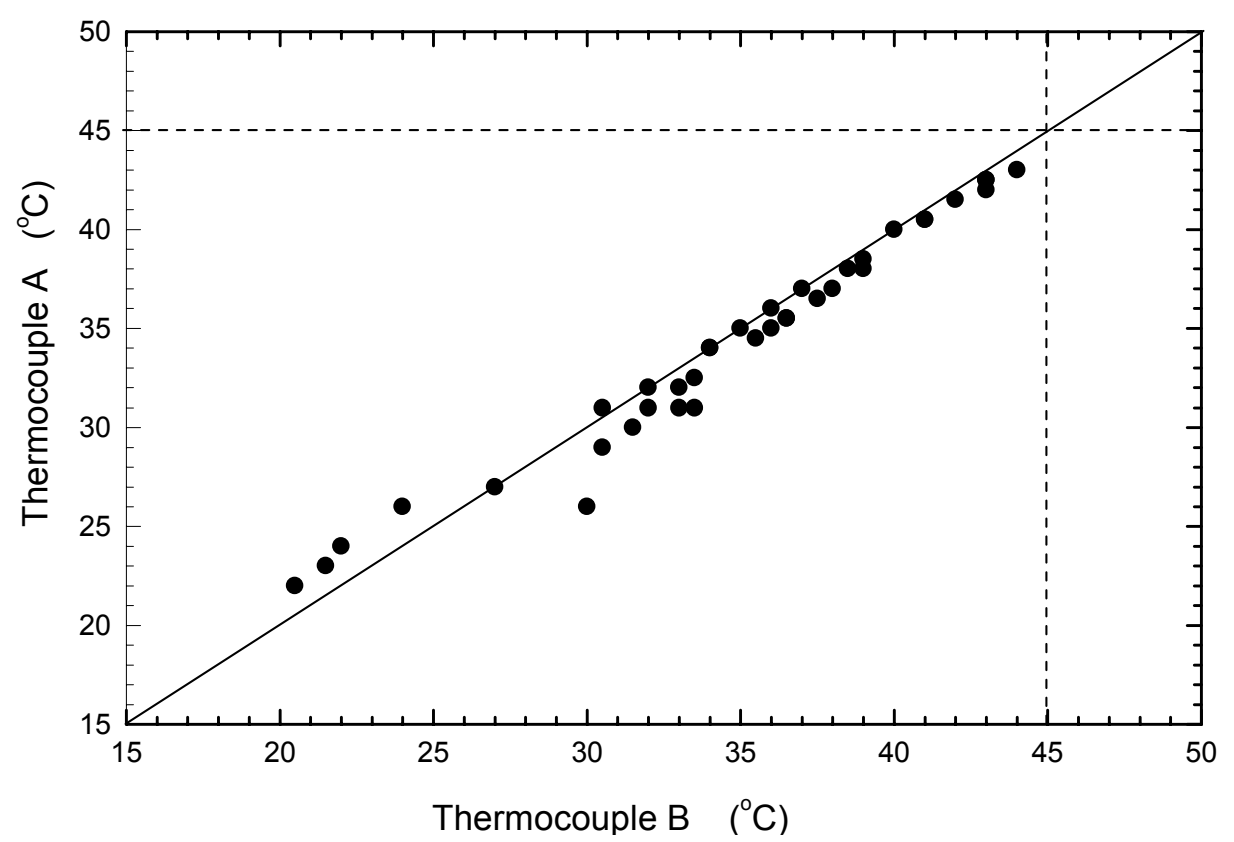

Fig. 1. Water temperature measurements in the reactor pool. Thermocouple A is located at the core inlet and thermocouple B is located at the opposite side of the pool. The dashed lines show the operating limit.

In line with the core model assumed in PARET, the core is subdivided in a number of regions over its radial cross section, each represented by a single coolant channel and associated fuel plate. Provision is made for weighting these regions for purposes of the reactivity feedback calculations. A description of the modelling approach used in the present simulations is given next.

Thermal-hydraulic modelling is based on the equations of conservation of mass, momentum and energy, formulated for a one-dimensional flow along the axial direction

$$
\begin{gathered}
\frac{\partial \bar{\rho}}{\partial t}=-\frac{\partial G}{\partial z} \\
\frac{\partial G}{\partial t}+\frac{\partial}{\partial z}\left(\frac{G^{2}}{\rho^{\prime}}\right)=-\frac{\mathrm{d} p}{\mathrm{~d} z}-\frac{f}{2 \bar{\rho} D_{e}}|G| G-\bar{\rho} g
\end{gathered}
$$




$$
\rho^{\prime \prime} \frac{\partial \bar{h}}{\partial t}+G \frac{\partial \bar{h}}{\partial z}=2 q^{\prime} / w_{c}
$$

where $t$ is time, $z$ the axial coordinate (positive upwards), $G$ the mass flow rate, $\mathrm{d} p / \mathrm{d} z$ the pressure gradient, $f$ the friction factor, $D_{e}$ the equivalent hydraulic diameter, $g$ the acceleration due to gravity, $q^{\prime}$ the heat flux, and $w_{c}$ the water channel width. The coolant may range between a subcooled liquid and a saturated two-phase mixture. Accordingly, the average coolant density $\bar{\rho}$ in the mass conservation equation, the average coolant enthalpy $\bar{h}$ in the energy equation, and the effective densities $\rho^{\prime}$ and $\rho^{\prime \prime}$ pertaining to the momentum equation and energy equation respectively, are defined as

$$
\begin{gathered}
\bar{\rho}=\rho_{l}(1-\alpha)+\rho_{v} \alpha \\
\bar{h}=h_{l}(1-\chi)+h_{v} \chi \\
\frac{1}{\rho^{\prime}}=\frac{1-\chi^{2}}{1-\alpha} \frac{1}{\rho_{l}}+\frac{\chi^{2}}{\alpha} \frac{1}{\rho_{v}} \\
\rho^{\prime \prime}=\left[\rho_{l} \chi+\rho_{v}(1-\chi)\right] \frac{\mathrm{d} \alpha}{\mathrm{d} \chi}
\end{gathered}
$$

where subscripts $l$ and $v$ denote liquid water and water vapor respectively, $\rho$ is density, $h$ enthalpy, $\alpha$ void fraction, and $\chi$ mass quality. In PARET, the two-phase friction factor and the void-to-quality relationship are determined using the Martinelli method (see, for example, in Collier, 1972). As indicated by momentum Eq. (2), both downflow and upflow conditions can be accommodated. Therefore, the model allows for a LOFA simulation throughout the whole transient, encompassing the decay phase of the downward flow, the flow reversal, and the establishment of the upward natural circulation.

Heat transfer in the fuel plates is determined from the solution of the one-dimensional conduction equation in the radial direction

$$
\frac{\partial}{\partial t}\left(\rho c_{p} T\right)=\frac{\partial}{\partial x}\left(k \frac{\partial T}{\partial x}\right)+f_{S} S
$$

where $T$ is temperature, $x$ the radial coordinate (normal to $z$ ), $\rho c_{p}$ the volumetric heat capacity, $k$ the thermal conductivity, $S$ the heat source per unit volume, and $f_{S}$ a heat source flag such that $f_{S}=1$ in the fuel meat and $f_{S}=0$ in the clad. At the clad-coolant interface, the convection boundary condition is applied. Heat transfer may take place by natural or forced convection, nucleate, transition, or fully-developed boiling, depending on the regime predicted. PARET supports a selection of heat transfer correlations in estimating convective heat transfer to coolant. In the present simulations we used the Dittus-Boelter correlation for turbulent-flow heat transfer, the McAdams correlation for two-phase heat transfer, and the Bergles-Rohsenow scheme for the transition from single to two-phase regime. Details on the previous correlations can be found in Collier (1972). A separate sensitivity study ascertained that the simulation results are not sensibly dependant on the choice of heat transfer correlations. For the laminar-flow regime we used a Nusselt number $\mathrm{Nu}=7.5$, with allowance for entrance effects. This selection is based on available analytical solutions for heat transfer in rectangular ducts (Shah and London, 1978).

In accordance with the point reactor model, the heat source per unit volume is assumed to be a separable function of space and time, determined in each plate from the average core power density $P(t)$ and appropriate axial $\left(F_{z}\right)$ and radial $\left(F_{x}\right)$ buckling parameters

$$
S=F_{z} F_{x} P(t) .
$$


Axial parameter $F_{z}$ is a chopped cosine function having a peak value such that

$($ peak power in the plate $) /($ average power in the plate $)=1.428$.

The specified value of 1.428, adapted from GRR-1 neutronic calculations, is consistent with the typical value of 1.5 considered in the IAEA benchmark exercise (IAEA, 1992). On the other hand, radial parameter $F_{x}$ is specified as

$$
F_{x}=(\text { average power in the plate }) /(\text { average power in the core })
$$

The average core power density $P(t)$ is calculated from a point reactor kinetics model with six groups of delayed neutrons

$$
\begin{gathered}
\frac{\mathrm{d} P}{\mathrm{~d} t}=\frac{\rho(t)-\beta}{\Lambda} P+\sum_{i=1}^{6} \lambda_{i} C_{i} \\
\frac{\mathrm{d} C_{i}}{\mathrm{~d} t}=\frac{\beta_{i}}{\Lambda} P-\lambda_{i} C_{i}, i=1,2, \ldots 6
\end{gathered}
$$

where $\rho(t)$ is the reactivity, $\lambda_{i}$ the precursor decay constant of the $i$ th group, $\beta_{i}$ the yield of the $i$ th group, $\beta$ the total delayed neutron fraction, $\beta=\sum \beta_{i}, \Lambda$ the mean neutron generation time, and $C_{i}$ the precursors number of the $i$ th group (times a units conversion constant). Reactivity $\rho(t)$ is determined by calculating continuous reactor feedback as follows

$$
\rho(t)=\rho_{e x}(t)+\sum_{j} a_{M} \chi_{j} \delta \bar{\rho}_{j}-\sum_{j} a_{T} \chi_{j} \delta T_{c, j}-\sum_{k} a_{F} \psi \psi_{k} \delta T_{F, k}
$$

In the above expression, the first term in the r.h.s. is the externally introduced reactivity (for example, the shutdown reactivity if scram is enabled) and the remaining terms are the various feedback contributions. As can be seen, three feedback mechanisms are taken into consideration, namely, changes of coolant density or void $\delta \bar{\rho}$, changes of coolant temperature $\delta T_{c}$ (spectrum effects only), and changes of fuel temperature $\delta T_{F}$ (Doppler effects). The corresponding coefficients of reactivity are respectively $a_{M}, a_{T}$ and $a_{F}$. The total reactivity feedback is calculated as the volume-weighted sum through all computational nodes, i.e. the whole core. Accordingly, $\chi_{j}$ is the volume fraction in the core of the $j$ th coolant computational node and $\psi_{k}$ the volume fraction in the core of the $k$ th fuel computational node. The approach is consistent with recent theoretical developments on the estimation of the moderator temperature coefficient in PWRs (Antonopoulos-Domis and Housiadas, 1999; Housiadas and Antonopoulos-Domis, 1999).

The simulations have been performed by dividing the core into two regions, i.e. assuming two parallel cooling channels and the associated fuel plates. The first channel represents the hottest channel in the core and the second represents the remainder of the core, hence, average core conditions. Previous simulation results have confirmed that a two-region description is sufficient for an accurate prediction of the core response in research reactor transients (IAEA, 1992). For the average channel and plate it is apparent that $F_{x}=1$, whereas for the hot channel and plate parameter $F_{x}$ is obtained as $F_{x}=F_{P} / 1.428$ where $F_{P}$ is the total peaking factor. The latter, defined as

$$
F_{P}=(\text { maximum power in the core }) /(\text { average power in the core }) \text {, }
$$

has been determined from available GRR-1 neutronic data to be $F_{P}=2.1$. This value compares well with the peaking factors usually calculated in research reactors (IAEA, 1992). The hot channel and associated hot plate are assigned with a feedback weight equal to $1 / N_{C H N}$ where $N_{C H N}$ is the total number of channels in the core 
( $N_{\text {CHN }}=554$, cf. Table 1), while the average channel and associated average plate are assigned with a weight equal to $1-1 / N_{C H N}$.

PARET employs a powerful numerical method for the calculation of the thermal-hydraulics, based on a finite differences scheme enhanced with a modified momentum integrated model (Woodruff, 1984). For the solution of the point kinetics a modified Runge-Kutta method is used. In the present simulations, good accuracy was obtained by using a time step of $50 \mathrm{~ms}$, along with 21 axial nodes for the solution of Eqs. (1-3) and 7 radial nodes (5 in the meat and 2 in the clad) for the solution of Eq. (8).

The simulations are performed in the following way. First, a steady state calculation is made at initial power level $P_{0}$, initial flow-rate $G_{0}$ (downward), and core inlet temperature $T_{\text {inlet }}=T_{0}$ where $T_{0}$ is the initial pool temperature. With the reactor thermal-hydraulics at $t=0$ determined, the transient run is forced by prescribing a flow-rate variation as

$$
G=G_{0} \exp (-t / \tau)
$$

where $\tau$ is a time constant describing the loss-of-flow decay. Provision is made to account for pool heating, considering that after a loss-of-flow event reactor power cannot be discharged to the heat exchanger. The original version of PARET assumes a constant core inlet temperature. A customized version of the code was devised, in which core inlet temperature is determined from a stationary heat balance over the pool volume. Accordingly, core inlet temperature is prescribed to vary as

$$
T_{\text {inlet }}=T_{0}+\frac{\int_{0}^{t} V_{\text {fuel }} P\left(t^{\prime}\right) \mathrm{d} t^{\prime}}{\rho_{c} c_{p c} V_{\text {pool }}}
$$

where $\rho_{c} c_{p c}$ is the volumetric heat capacity of the coolant (water), $V_{\text {fuel }}$ the fuel volume, and $V_{\text {pool }}$ the volume of the pool. Equation (17) implies that uniform temperature conditions prevail in the pool. This is a reasonable assumption considering the temperature results shown in Fig. 1, as previously discussed. At $t=2 \tau$, i.e. when flow-rate $G$ reduces to $\approx 15 \%$ of the initial value $G_{0}$, the flapper valve sealing the lower plenum is assumed to open, hence, downward forced flow stops and natural circulation develops through flow reversal. This is the most adverse part of the transient because the hot coolant of the lower plenum will first undertake natural cooling, until cool water from the pool expels progressively the hot lower plenum volume. This phase is simulated as follows: inlet coolant temperature $T_{\text {inlet }}$ is forced to jump to the value of the exit coolant temperature predicted at $t=2 \tau$, and then is forced to match linearly Eq. (17) over a period of $5 \mathrm{~s}$.

The threshold conditions for flow instability are determined using the developments of Whittle and Forgan (1967). The latter proposed a correlation for the Onset of Flow Instability (OFI) point in terms of a critical value for the ratio

$$
R=\frac{T_{\text {out }}-T_{\text {inlet }}}{T_{\text {sat }}-T_{\text {inlet }}}
$$

as follows

$$
R_{\mathrm{OFI}}=\frac{1}{1+25 \frac{D_{e}}{H}}
$$

where $H$ is the height of the channel and $T_{\text {out }}$ and $T_{\text {sat }}$ are the outlet and saturation temperatures respectively. The above correlation has been inferred from measurements in narrow rectangular channels at low-pressure conditions and proved to be the most satisfactory for research reactor applications (Hu and Bernard, 1999; IAEA, 1980; Mishima et al., 1984). 


\begin{tabular}{|c|c|}
\hline \multicolumn{2}{|l|}{ Kinetic data } \\
\hline$\Lambda(\mu \mathrm{s})$ & 57.6 \\
\hline$\beta$ & 0.0066 \\
\hline$\beta / \sum_{i} \frac{\beta_{i}}{\lambda_{i}}\left(\mathrm{~s}^{-1}\right)$ & 0.079 \\
\hline \multicolumn{2}{|l|}{ Peaking factors } \\
\hline Axial & 1.428 \\
\hline Radial & 1.471 \\
\hline$F_{P}(=$ Axial $\times$ Radial $)$ & 2.1 \\
\hline \multicolumn{2}{|l|}{ Feedback reactivity coefficients } \\
\hline$a_{M}\left(\$ / \mathrm{g} \mathrm{cm}^{-3}\right)$ & $35.799(=0.35799 \$ / \%$ void $)$ \\
\hline$a_{T}\left(\$ /{ }^{\circ} \mathrm{C}\right)$ & $1.341 \times 10^{-2}$ \\
\hline$a_{F}\left(\$ /{ }^{\circ} \mathrm{C}\right)$ & $2.370 \times 10^{-3}$ \\
\hline \multicolumn{2}{|l|}{ Clad thermal properties } \\
\hline$k\left(\mathrm{~W} \mathrm{~m}^{-1} \mathrm{~K}\right)$ & 217 \\
\hline$\rho c_{p}\left(\mathrm{~J} \mathrm{~m}^{-3} \mathrm{~K}\right)$ & $1242 T+2.07 \times 10^{6}(\mathrm{~T}$ in $\mathrm{K})$ \\
\hline \multicolumn{2}{|l|}{ Fuel thermal properties } \\
\hline$k\left(\mathrm{~W} \mathrm{~m}^{-1} \mathrm{~K}\right)$ & 142 \\
\hline$\rho c_{p}\left(\mathrm{~J} \mathrm{~m}^{-3} \mathrm{~K}\right)$ & $925 T+2.03 \times 10^{6}(\mathrm{~T}$ in $\mathrm{K})$ \\
\hline
\end{tabular}

The reactor data and the material thermal properties used in the simulations are summarized in Table 2. As mentioned before, the kinetic data, peaking factors, and reactivity feedback coefficients have been adapted from available neutronic analysis results. The used input parameters are similar to those usually employed in research reactor simulations (Nasir et al., 1999; IAEA, 1992; Woodruff, 1984), hence, the obtained results will be typical of such type of reactors.

\section{Results}

It is well known that the basic system variables determining the two-phase flow stability boundaries are heat flux, flow-rate and inlet subcooling. Hence, a parametric analysis with respect to initial reactor power $P_{0}$, peaking factor $F_{P}$, flow-rate decay constant $\tau$, and initial pool temperature $T_{0}$ seems to be in order. Subsequently, we give the results of the analysis, as obtained for the case of the hot channel.

The effect of the initial pool temperature $T_{0}$ is first addressed. Figure 2 shows the responses of power (Fig. 2a) and clad and coolant temperatures (Fig. 2b) for $T_{0}=40^{\circ} \mathrm{C}$ (case 1) and $T_{0}=25^{\circ} \mathrm{C}$ (case 2). For comparison, the results from a simulation with $T_{0}=40^{\circ} \mathrm{C}$ and reactor scram enabled (case 3) are also shown. The calculations are performed using the reference input data of Tables 1 and 2, along with a flow-decay time constant of $\tau=25 \mathrm{~s}$, thus simulating a slow transient. In case 3, the reactor shutdown is simulated by specifying as $\rho_{e x}$ in Eq. (14) the total worth (negative) of the control rods. The shutdown reactivity insertion is assumed to take place when flowrate attains the low-limit trip point, presumed at $50 \%$ of the nominal flow-rate. As can be seen, the rates of power decrease and temperature rise are practically the same in all three cases. In cases 1 and 2, the power profiles show clearly the strong feedback effects. Obviously, in the shutdown case (case 3), reactor power, as well as temperature, drop immediately after scram is actuated. At $t=50 \mathrm{~s}(=2 \tau)$ flow reversal takes place, accompanied by a marked increase in temperature because of the re-circulation through the core of the hot water of the lower plenum. For the unprotected cases 1 and 2 the temperature rise takes the form of a sharp peak, which, in turn, induces a deep in the reactor power profile. This is the most crucial part of the transient. As shown in Fig. 2b, clad temperature (solid lines) may well (case 1) or may not (case 2) exceed the Onset of Nucleate Boiling (ONB) point, depending on the initial temperature level $T_{0}$. In case 1 significant boiling takes place, whereas in case 2 no significant voiding is produced in the channel but only, at worse, some minor subcooled boiling. This is clearly depicted by the coolant temperature (dashed lines), which in case 1 reaches saturation, whereas in case 2 remains always highly subcooled. The ratios $R / R_{\mathrm{OFI}}$ are compared in Fig. 3. As can be seen, in case 1 the ratio becomes 

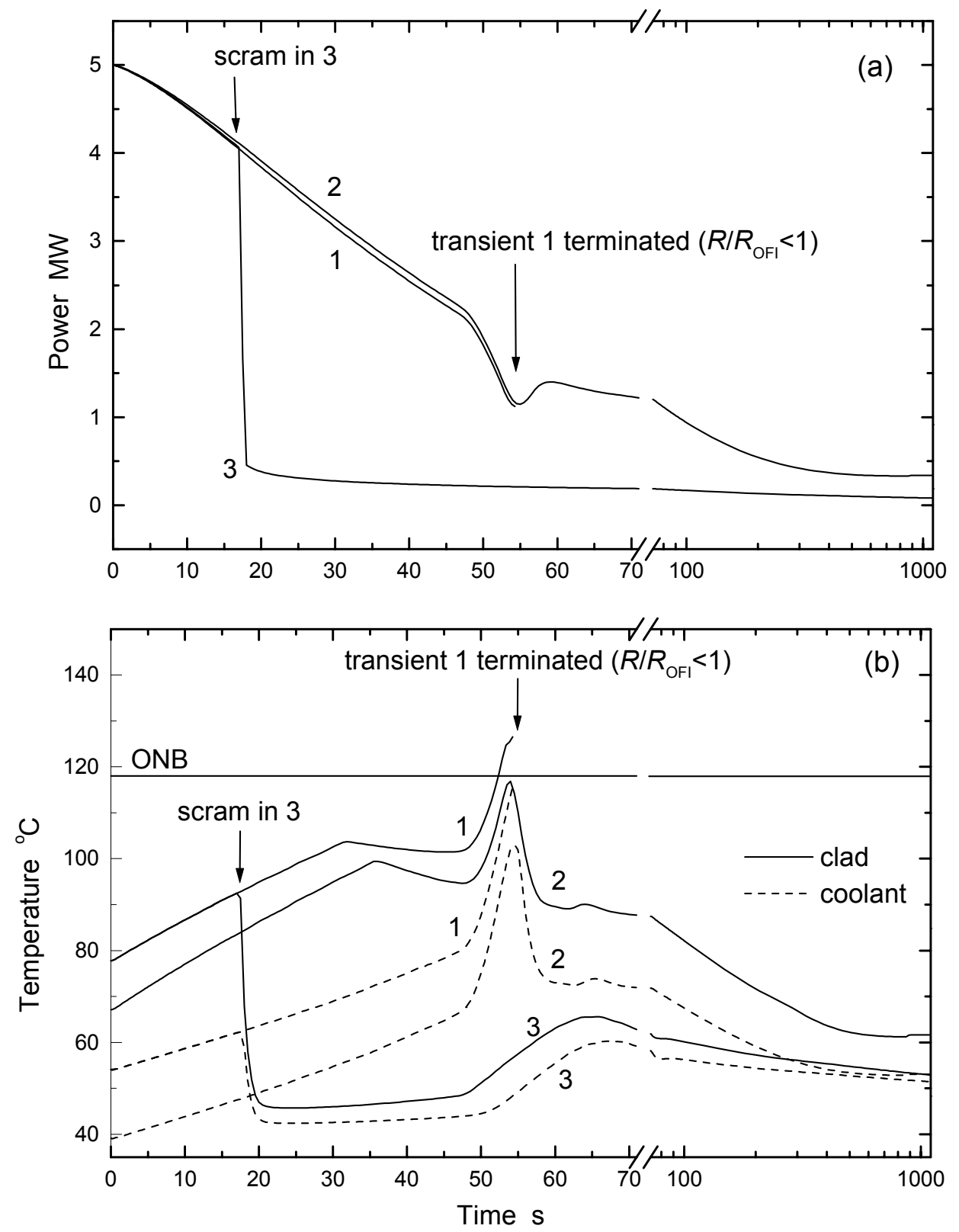

Fig. 2. Responses of power (a) and clad and coolant temperatures (b) for the three cases considered. $1: T_{0}=40^{\circ} \mathrm{C}$, scram disabled; 2: $T_{0}=25^{\circ} \mathrm{C}$, scram disabled; $3: T_{0}=40^{\circ} \mathrm{C}$, scram enabled.

less than one, hence, inception of flow instability is predicted. In contrast, in case $2, R / R_{\mathrm{OFI}}$ remains always above one, thus, stability is maintained throughout the transient and a new equilibrium state is ultimately established. The reactor power reduces constantly due to feedback and is found stabilised at a level corresponding to less then $10 \%$ of the initial power. At such power levels natural convection is sufficient to cool the core. In the shutdown case (case 3 ), the ratio $R / R_{\mathrm{OFI}}$ remains always, as expected, well within the stable region. In case 1 the course of the transient is not predicted further. Calculation of flow instabilities is beyond the scope of the present investigation. This would require a full two-phase modelling approach, with an accurate calculation of transient pressures and flow fluctuations. PARET code is limited in this respect, for it employs incompressible hydrodynamics and simplified void generation equations.

Figure 4 shows the calculated boundary between stable and unstable regions. The boundary is described in terms of maximum initial pool temperature $T_{0}$ as function of the initial reactor power $P_{0}$ and parameterized by the peaking factor $F_{P}$ and the decay constant $\tau$. The parameters are let to vary over enlarged ranges to cover the usual research reactor cases, as considered in IAEA (1992). More specifically, $P_{0}$ is varied between 2 and 10 MW. $F_{P}$ is changed stepwise between 1.9 (moderate buckling) and 2.5 (large peaking accommodating possible engineering hot channel factors). Finally, $\tau$ is either $1 \mathrm{~s}$ corresponding to a fast transient (e.g. valve failure) or 25 s corresponding to a slow transient (e.g. pump failure with large flow inertia). As the results of Fig. 4 indicate, all parameters influence significantly the stability boundary, confirming thus the important role played by heat flux, 


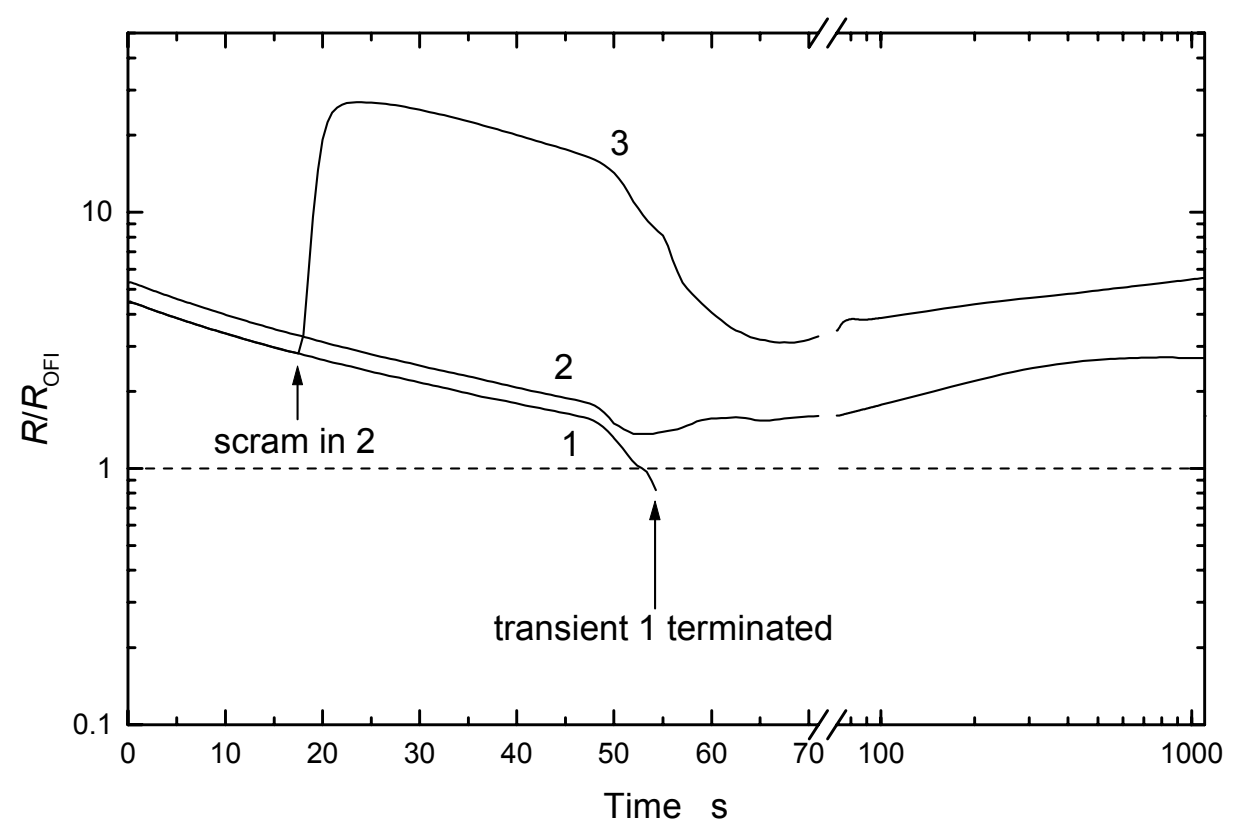

Fig. 3. Evolution of the ratio $R / R_{\mathrm{OFI}}$ as function of time for the three cases considered (as in Fig. 2).

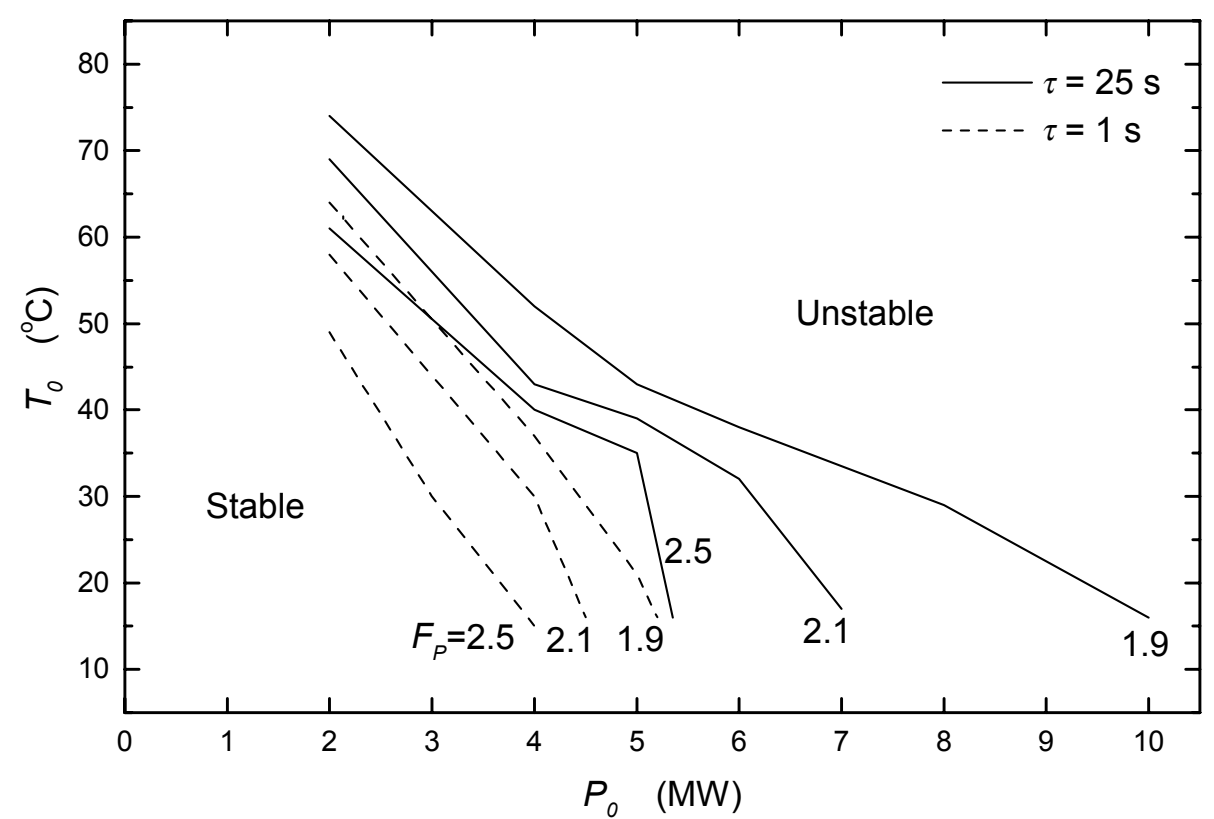

Fig. 4. Boundaries between stable and unstable regions in terms of limit $T_{0}$ as function of $P_{0}$, with $F_{P}$ and $\tau$ as parameters.

flow-rate and subcooling. The following trends are observed. As power level increases the maximum tolerable pool temperature decreases, i.e. higher subcooling is required to sustain stable conditions. With increasing $F_{P}$ i.e. higher power density in the hot channel, the boundary moves to the right, thereby the stability region shrinks. Similarly, with small $\tau$ flow-rate is reduced rapidly and a smaller stability region is delineated. The above trends are consistent with those observed in a recent experimental investigation (Oh and Chapman, 1996). The chart of Fig. 4 provides useful engineering guidance in assessing a LOFA sequence or, eventually, specifying operational limits. For instance, for the case of GRR-1 $\left(P_{0}=5 \mathrm{MW}, F_{P}=2.1\right)$ a primary pump failure with slow coast-down ( $\tau=25 \mathrm{~s}$ ) would not imply, even without scram, any consequence for the core if initial pool temperature is below $38^{\circ} \mathrm{C}$. Instead, in case of an inadvertent rapid valve closing ( $\left.\tau=1 \mathrm{~s}\right)$ emergency shutdown is necessary, otherwise core integrity will be compromised, no matter what are the initial reactor conditions.

The sensitivity of the results to variations in kinetic parameters, reactivity coefficients and fuel thermal properties has been considered. The above parameters have been changed in a way to bracket the variations observed in the literature among different research reactor cores, in particular, to cover the transition core configurations (mixed LEU/HEU). The results are shown in Table 3 in terms of limit pool temperature for stable 
Table 3. Sensitivity of results to variations in kinetic parameters, fuel thermal properties and reactivity coefficients $\left(P_{0}=6 \mathrm{MW}, F_{P}=2.1, \tau=25 \mathrm{~s}\right)$

\begin{tabular}{cccc}
\hline Parameter & Variations & Comments & $T_{0}\left({ }^{\circ} \mathrm{C}\right)$ \\
\hline$\Lambda(\mu \mathrm{s})$ & 42 & Nasir et al., 1999 & 32 \\
& 57.6 & reference value & 32 \\
\hline \multirow{2}{*}{$\beta$} & 0.0066 & reference value & 32 \\
& 0.0073 & Woodruff, 1984 & 32 \\
\hline \multirow{2}{*}{$k$, fuel } & 50 & IAEA, 1992 & 32 \\
$\left(\mathrm{~W} \mathrm{~m}{ }^{-1} \mathrm{~K}\right)$ & 142 & reference value & 32 \\
\hline \multirow{2}{*}{$a_{M}$} & 160 & Nasir et al., 1999 & 33 \\
$(\$ / \%$ void $)$ & 0.28639 & $-20 \%$ change & 29 \\
& 0.35799 & reference value & 32 \\
$a_{T}$ & 1.42959 & $+20 \%$ change & 34 \\
$\left(\$ /{ }^{\circ} \mathrm{C}\right)$ & $1.341 \times 10^{-2}$ & $-20 \%$ change & 30 \\
\hline \multirow{2}{*}{$a_{F}$} & $1.609 \times 10^{-2}$ & reference value & 32 \\
$\left(\$ /{ }^{\circ} \mathrm{C}\right)$ & $3.6 \times 10^{-5}$ & $+20 \%$ change & 36 \\
& $1.42 \times 10^{-3}$ & HEU fuel & 26 \\
& $2.37 \times 10^{-3}$ & $-40 \%$ change & 29 \\
\hline
\end{tabular}

conditions. Only the parameter indicated was changed in each case, while the others were kept at their reference values given in Table 2. As can be seen, the kinetic parameters $\Lambda$ and $\beta$ have no influence on the results. Similarly, the uncertainty in the thermal conductivity of the fuel, though large, does not have an impact on the estimation of the stability limit. As expected, the reactivity coefficients have a measurable effect on the results because they determine the strength of reactor feedback. Still, however, their impact is rather restrained and would not alter significantly the results of Fig. 5. A notable exception is the case of the Doppler coefficient. The latter is the only parameter among the considered ones that changes by orders of magnitude in the HEU to LEU transition. In the LEU fuel, because of the abundance of the contained ${ }^{238} \mathrm{U}$, the Doppler coefficient is important, inducing strong reactor feedback. Therefore, markedly wider stability margins are obtained with LEU, as clearly indicated by the results of Table 3. This finding is another demonstration of the safety enhancement achieved with the use of LEU fuel in research reactors.

\section{Conclusions}

A method is presented that enables the analysis of loss-of-flow sequences, with scram disabled, in pool-type research reactors. The analysis is based on simulations performed with a customized version of the well-known coupled neutronics, thermal-hydraulics code PARET. The employed modelling approach is described in detail and the used assumptions thoroughly discussed.

The obtained results can be regarded as typical of pool-type research reactors. Although GRR-1 reactor was used as a basis, care was exercised in the selection of parameters and ranges of variation, so to cover the usual research reactor cases.

The simulations provided with a deep understanding of the mechanisms that determine the course of a loss-offlow transient. The general physical picture obtained is the following: as flow-rate is falling and reactor remains unprotected, coolant and fuel temperatures rise. The increasing temperatures induce a negative reactivity feedback and a corresponding power decrease. Eventually, reactor power is lowered enough, so that natural convection is sufficient to cool the core. In this case an equilibrium situation is re-established in the reactor and the transient terminates without causing core damage. However, the safe transition from the initial to the final equilibrium state is interrupted if an unstable regime is encountered. This is connected with the development of massive bulk boiling in the hot channel, inducing flow instabilities of the Ledinegg type.

The stability boundaries have been determined in terms of initial reactor power, initial pool temperature, peaking factor, and flow-decay time constant. A useful chart is developed (Fig. 4), which may provide engineering guidance in assessing unprotected loss-of-flow sequences and, eventually, specifying safety limits. 


\section{References}

Achard, J. L., Drew, D. A., Lahey, R. T., 1985. The analysis of nonlinear density-wave oscillations in boiling channels. Journal of Fluid Mechanics 155, 213-232.

Antonopoulos-Domis, M., Housiadas, C., 1999. Moderator temperature coefficient of reactivity in Pressurized Water Reactors: theoretical investigation and numerical simulations. Nuclear Science and Engineering 132, 337-345.

Collier, J. G., 1972. Convective boiling and condensation. McGraw-Hill, London.

Housiadas, C., Antonopoulos-Domis, M., 1999. The effect of fuel temperature on the estimation of the moderator temperature coefficient in PWRs. Annals of Nuclear Energy 26, 1395-1405.

Hu, L. W., Bernard, J. A., 1999. Thermal-hydraulic criteria for the MIT research reactor safety limits calculation. Transactions of the American Nuclear Society 81, 114-115.

IAEA, 1980. Research reactor core conversion from use of Highly Enriched Uranium to the use of Low Enriched Uranium fuels guidebook. International Atomic Energy Agency, Report IAEA-TECDOC-233.

IAEA, 1986. Probabilistic safety assessment for research reactors. International Atomic Energy Agency, Report IAEA-TECDOC-400.

IAEA, 1992. Research reactor core conversion guidebook. International Atomic Energy Agency, Report IAEATECDOC-643.

IAEA, 1994. Safety assessment of research reactors and preparation of the safety analysis report. International Atomic Energy Agency, Report IAEA-SAFETY SERIES No 35-G1.

Mirza, A. M., Khanam, S., Mirza, N. M., 1998. Simulation of reactivity transients in current MTRs. Annals of Nuclear Energy 25, 1465-1484.

Mishima, K., Kanda, K., Shibata, T., 1984. Thermal-hydraulic analysis for core conversion to the use of lowenriched uranium fuels in the KUR research reactor. Kyoto University, Report KURRI-TR-258.

Nasir, R., Mirza, N. M., Mirza, S. M., 1999. Sensitivity of reactivity insertion limits with respect to safety parameters in a typical MTR. Annals of Nuclear Energy 26, 1517-1535.

Obenchain, C. F., 1969. PARET-A program for the analysis of reactor transients. Idaho National Engineering Laboratory, Report IDO-17282.

Oh, C. H., Chapman, J. C., 1996. Two-phase flow instability for low-flow boiling in vertical uniformly heated thin rectangular channels. Nuclear Technology 113, 327-337.

Saha, P., Ishii, M., Zuber, N., 1976. An experimental investigation of the thermally induced flow oscillations in two-phase systems. Journal of Heat Transfer 98, 616-622.

Shah, R. K., London, A. L., 1978. Laminar flow forced convection in ducts. Academic Press, New York.

Whittle, R. H., Forgan, R., 1967. A correlation for the minima in the pressure drop versus flow rate curves for subcooled water flowing in narrow heated channel. Nuclear Engineering and Design 6, 89-99.

Woodruff, W. L., 1984. A kinetics and thermal-hydraulics capability for the analysis of research reactors. Nuclear Technology 64, 196-206.

Yadigaroglu, G., Bergles, A. E., 1972. Fundamental and higher-mode density-wave oscillations in two-phase flow. Journal of Heat Transfer 94, 189-195. 